$03 ; 12$

\title{
Критическая напряженность электрического поля для анизотропного спинодального распада в воде
}

\author{
() Д.И. Карпов ${ }^{1,2}$, Д.А. Медведев ${ }^{1,2}$, А.Л. Куперштох ${ }^{1,2}$ \\ ${ }^{1}$ Институт гидродинамики им. М.А. Лаврентьева СО РАН, Новосибирск \\ ${ }^{2}$ Новосибирский государственный университет \\ E-mail: karpov@hydro.nsc.ru
}

Поступило в Редакцию 1 декабря 2016 г.

Выполнены расчеты пороговой напряженности электрического поля для анизотропного спинодального распада воды в сверхкритической области при $T=670$ и $350 \mathrm{~K}$. Пороговая напряженность зависит от второй производной диэлектрической проницаемости по плотности. Зависимости диэлектрической проницаемости от плотности получены методом флуктуаций вектора поляризации вещества в широком диапазоне плотностей при указанных температурах. Временнб́е ряды для флуктуаций вектора поляризации ансамблей молекул воды построены на основе молекулярно-динамических расчетов.

DOI: 10.21883/PJTF.2017.16.44928.16590

Под действием экстремально сильного электрического поля термодинамическое состояние диэлектрической жидкости (плотного газа) может при определенных условиях стать неустойчивым, в результате чего происходит фазовый переход с образованием газовой фазы в жидкости (жидкой фазы в паре). В [1] было показано, что смещение по температуре и давлению критической точки диэлектрической жидкости в электрическом поле пропорционально квадрату напряженности поля и второй производной диэлектрической проницаемости вещества по плотности. Также происходит смещение всей спинодали. Это означает, что устойчивые состояния, которые были достаточно близки к бинодали, могут оказаться в области метастабильных состояний или даже в области неустойчивости. В работе [2] было показано, что распад на жидкую и газовую фазы в сильном электрическом поле происходит анизотропно, т.е. в жидкости формируются паровые каналы, которые имеют вытянутую форму и ориентированы вдоль линий напряженности 
поля. Также был вычислен инкремент неустойчивости и показано, что он зависит от второй производной диэлектрической проницаемости вещества по плотности. Это явление, в частности, может объяснить, каким образом при электрическом пробое жидкого диэлектрика за очень малое время ( $1 \mathrm{~ns})$ возникают стримерные каналы, в которых плотность вещества на порядки величины меньше плотности жидкости. Электрическая прочность вещества в каналах на порядки меньше, чем в самой жидкости, что приводит к развитию в них частичных разрядов и формированию стримеров в жидком диэлектрике.

Для оценки минимальной критической напряженности поля, при которой возникает анизотропный распад, необходимо знать зависимость диэлектрической проницаемости вещества от плотности $\varepsilon(\rho)$ в достаточно широком диапазоне плотностей при постоянной температуре. Таких измерений в литературе очень мало, что видно, например, из обзора [3]. Задача настоящей работы - найти зависимости $\varepsilon(\rho)$ исходя из микроскопического рассмотрения ансамбля модельных молекул воды и определить критические напряженности электрического поля, необходимые для анизотропного спинодального распада [1]. В работе выполнены расчеты диэлектрической проницаемости воды при температуре $T=670 \mathrm{~K}$, которая выше критической температуры для воды, и при двух значениях температуры ниже критической: $T=350$ и $300 \mathrm{~K}$.

Использовался метод расчета диэлектрической проницаемости через флуктуации вектора поляризации вещества [4]. Для получения значений вектора поляризации в разные моменты времени рассчитывалась эволюция ансамблей из 5000 молекул воды методом молекулярной динамики. Использовался пакет LAMMPS [5], начальное состояние ансамбля молекул воды генерировалось с помощью пакета moltemplate [6]. Моделирование выполнялось для $N-V-T$-ансамбля с условиями зеркального отражения на границах. Предварительно ансамбль термализовался. Расчеты выполнялись на кластере Информационно-вычислительного центра Новосибирского государственного университета [7], что позволило существенно сократить время вычислений.

Для описания свойств воды использовалась модель молекулы $\mathrm{SPC} / \mathrm{E}[8]$. Для учета электростатических взаимодействий в центры атомов водорода и кислорода помещаются три точечных электрических заряда. При этом электрический заряд кислорода равен -0.84 единиц элементарного заряда, заряд водорода равен 0.42 единиц элементарного заряда. Расстояние между атомами водорода и кислорода было фиксиро-

Письма в ЖТФ, 2017, том 43, вып. 16 


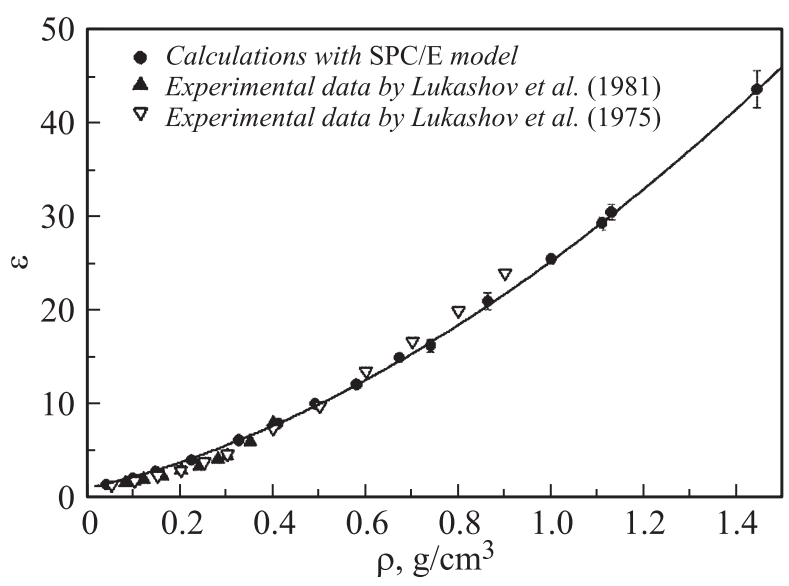

Рис. 1. Зависимость диэлектрической проницаемости воды от плотности на изотерме $T=670 \mathrm{~K}$. Кружки - результаты расчета, кривая — аппроксимация (1), треугольники - экспериментальные данные Лукашова с коллегами (взяты из обзора [3]).

вано и равно $0.1 \mathrm{~nm}$, угол между связями фиксирован и равен $109.47^{\circ}$. Согласно [9], такая модель молекулы воды точнее описывает кривые сосуществования жидкости и пара в области температур от $300 \mathrm{~K}$ и выше, чем ряд других моделей (TIP4P, TIP5P, Dang-Chang). Это является существенным в случае, когда необходимо описывать эволюцию двухфазных сред или фазовые переходы.

Через равные промежутки времени, соответствующие физическому времени 5 ps, фиксировались текущие состояния всех молекул воды. Далее по распределению электрических зарядов определялось текущее значение дипольного момента ансамбля. Полученный таким образом временной ряд использовался для расчета диэлектрической проницаемости согласно формуле [4]

$$
\varepsilon=1+\frac{\left(\left\langle\mathbf{M}^{2}\right\rangle-\langle\mathbf{M}\rangle^{2}\right)}{3 \varepsilon_{0} V k T} .
$$

Здесь М - дипольный момент ансамбля, $V$ - объем ансамбля, $T$ - температура, $k$ - постоянная Больцмана, $\varepsilon_{0}-$ диэлектрическая постоянная.

2 Письма в ЖТФ, 2017, том 43, вып. 16 


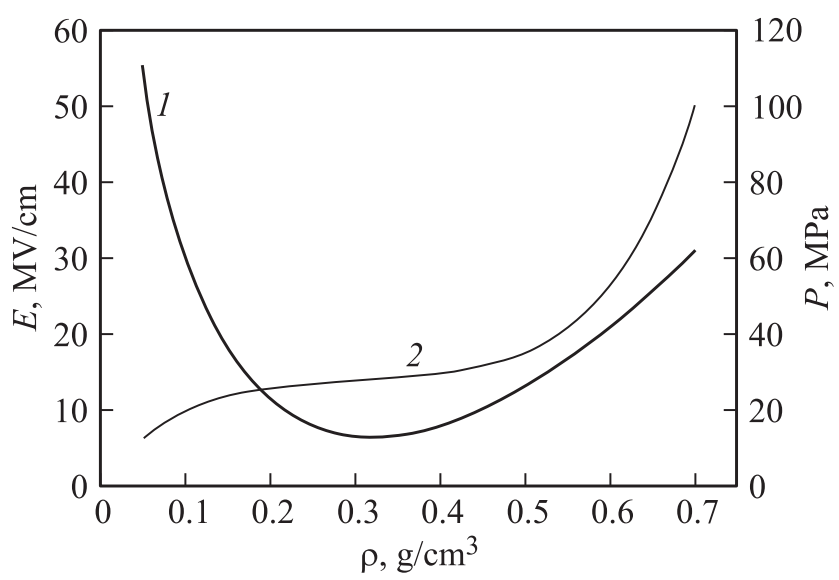

Рис. 2. Зависимость критического поля для анизотропного спинодального распада от плотности (1) и изотерма воды (2). $T=670 \mathrm{~K}$.

Выполнены расчеты диэлектрической проницаемости для диапазона плотностей от 0.039 до $1.44 \mathrm{~g} / \mathrm{cm}^{3}$ при температуре $T=670 \mathrm{~K}$. Эта температура соответствует надкритической области на диаграмме состояний (критическая точка воды $647 \mathrm{~K}$ ), что позволило получить значения $\varepsilon$ в очень широком диапазоне плотностей в той области параметров, где нет фазового перехода. Известно, что вариации $\varepsilon$ уменьшаются медленно с количеством элементов временно́го ряда М. Поэтому накопление данных выполнялось в течение больших промежутков времени $(\sim 10 \mathrm{~ns})$. При этом состояния ансамбля записывались через каждые 5 ps. Полученные в расчетах значения диэлектрической проницаемости при $T=670 \mathrm{~K}$ хорошо согласуются с данными экспериментов [3] (рис. 1).

Построены аппроксимации зависимости диэлектрической проницаемости от плотности при температуре $T=670$ К в диапазоне плотностей от 0.039 до $1.44 \mathrm{~g} / \mathrm{cm}^{3}$. Зависимость $\varepsilon(\rho)$ хорошо аппроксимируется выражением

$$
\varepsilon=1+11.25 \rho+14.05 \rho^{2}-0.98 \rho^{3},
$$

где плотность измеряется в единицах $\mathrm{g} / \mathrm{cm}^{3}$. 

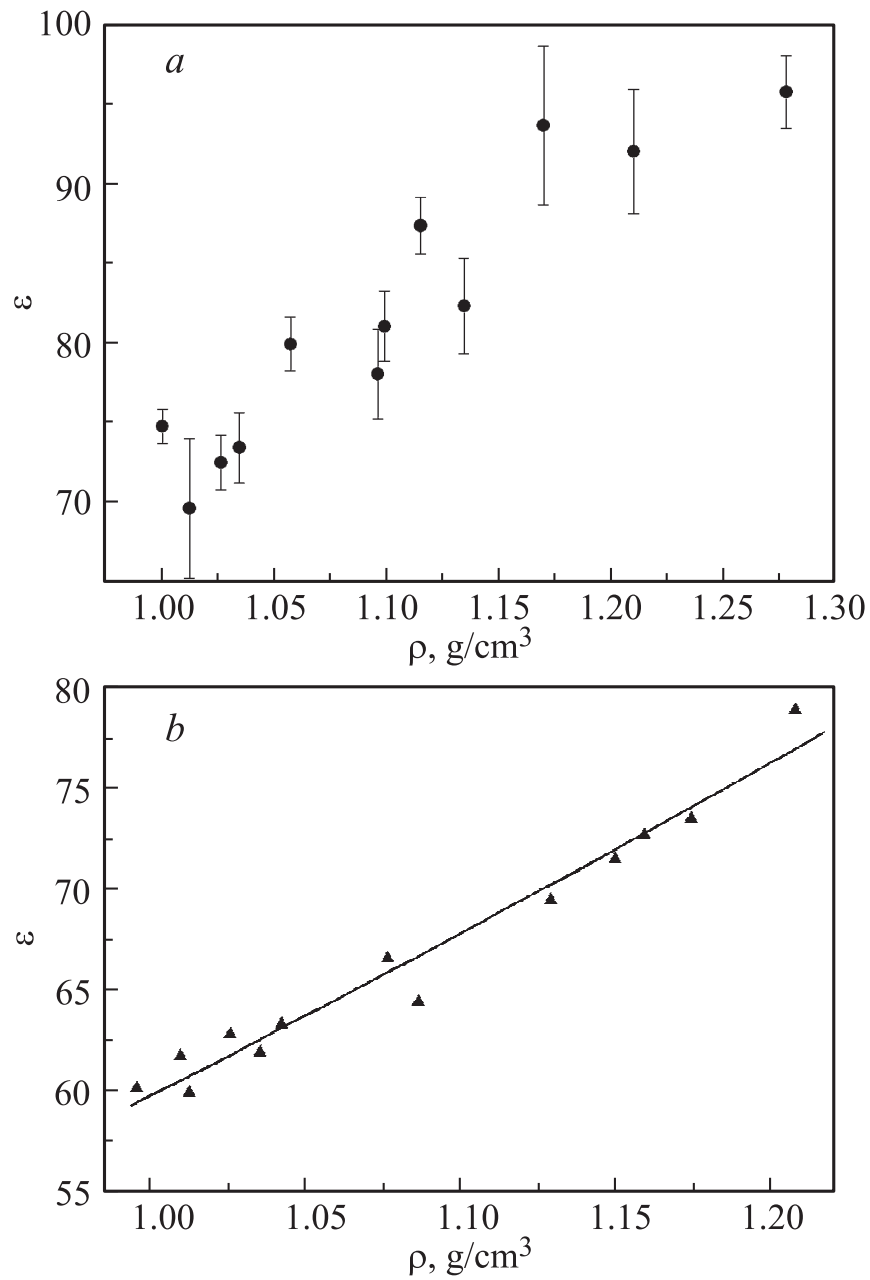

Рис. 3. Диэлектрическая проницаемость при низких температурах: $T=300(a)$ и $350 \mathrm{~K}(b)$. Кривая - аппроксимация (2) результатов расчетов (треугольники).

Критическое значение напряженности поля анизотропного спинодального распада (инициирования пробоя в жидкости) можно рассчи-

2* Письма в ЖТФ, 2017, том 43, вып. 16 
тать из соотношения [2]

$$
\left(\frac{\partial p}{\partial \rho}\right)_{T}=\frac{\varepsilon_{0} E_{0}^{2} \rho}{2}\left(\frac{\partial^{2} \varepsilon}{\partial \rho^{2}}\right)_{T} .
$$

Для вычисления производной $\left(\frac{\partial p}{\partial \rho}\right)_{T}$ использовалось реальное уравнение состояния воды „IAPWS Industrial Formulation 1997 for the thermodynamic properties of water and steam“ [10]. Полученные оценки критической напряженности поля $E_{0}$ для анизотропного спинодального распада приведены на рис. 2 (кривая 1). Кривая 2 на рис. 2 представляет изотерму воды $T=670 \mathrm{~K}$, вдоль которой рассчитаны значения $E_{0}$.

При относительно низких температурах $(T=300$ и $350 \mathrm{~K})$ наблюдался значительный разброс рассчитанных значений $\varepsilon$ (рис. $3, a$ ), что не позволило получить достаточно гладкую зависимость $\varepsilon(\rho)$. При $T=350 \mathrm{~K}$ удалось частично преодолеть эту сложность, увеличив время расчетов до $\sim 20 \mathrm{~ns}$. На рис. $3, b$ треугольниками показаны результаты расчетов $\varepsilon(\rho)$ при $T=350 \mathrm{~K}$ для модели молекулы с фиксированной длиной связей $\mathrm{O}-\mathrm{H}$. Данные хорошо аппроксимируются зависимостью (сплошная кривая)

$$
\varepsilon=1+31.36 \rho+22.54 \rho^{2}
$$

где плотность измеряется также в единицах $\mathrm{g} / \mathrm{cm}^{3}$. При этой температуре для плотности $1 \mathrm{~g} / \mathrm{cm}^{3}$ получено значение критической напряженности поля $E=23.9 \mathrm{MV} / \mathrm{cm}$. Это значение близко к оценкам величины напряженности поля инициирования импульсного электрического разряда в воде, полученным на основе известных экспериментальных данных.

Таким образом, получены зависимости диэлектрической проницаемости воды от плотности при различных температурах. Вычислены критические значения напряженности поля, необходимые для анизотропного спинодального распада воды в сильном электрическом поле, что дает возможность оценить электрическую прочность чистой воды.

Работа выполнена при финансовой поддержке Российского научного фонда (грант № 16-19-10229). Авторы благодарны Информационновычислительному центру Новосибирского государственного университета за предоставленные вычислительные ресурсы.

Письма в ЖТФ, 2017, том 43, вып. 16 


\section{Список литературы}

[1] Ландау Л.Д., Лифиии, Е.М. Электродинамика сплошных сред: теоретическая физика. М.: ГИФМЛ, 1959. 532 с.

[2] Kupershtokh A.L., Medvedev D.A. // Phys. Rev. E. 2006. V. 74. P. 021505 (1-5).

[3] Fernandes D.P., Mulev Y., Goodwin A.R.H., Levelt Sengers J.M.H. // J. Phys. Chem. Ref. Data. 1995. V. 24. N 1. P. 33-69.

[4] Pan D., Spanu L., Harrison B., Sverjensky D.A., Galli G. // PNAS. 2013. V. 110. N 17. P. 6646-6650.

[5] Large-scale Atomic/Molecular Massively Parallel Simulator; http://lammps.sandia.gov

[6] Moltemplate 1.34. http://www.moltemplate.org

[7] Информационно-вычислительный центр Новосибирского государственного университета. http://nusc.nsu.ru/

[8] Berendsen H.J.C., Grigera J.R., Straatsma T.P. // J. Phys. Chem. 1987. V. 91. P. 6269-6271.

[9] Yoo S., Zeng X.C. // J. Chem. Phys. 2002. V. 117. N 2. P. 9518-9519.

[10] The IAPWS industrial formulation 1997 for the thermodynamic properties of water and steam, Water/Steam Thermodynamic Surface on the WEB-site of Moscow Power Engineering Institute, Technical University; http://twt.mpei.ac.ru/MCS/Worksheets/WSP/VTPs.xmcd

Письма в ЖТФ, 2017, том 43, вып. 16 